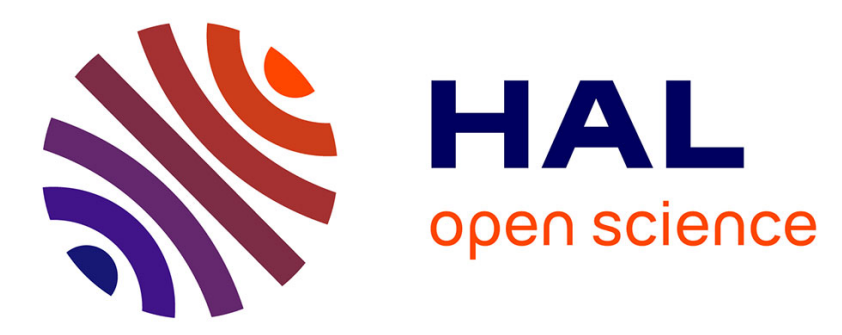

\title{
Computation of Saturation Dependence of Effective Diffusion Coefficient in Unsaturated Argillite Micro-fracture by Lattice Boltzmann Method
}

A. Genty, S. Gueddani, M. Dymitrowska

\section{- To cite this version:}

A. Genty, S. Gueddani, M. Dymitrowska. Computation of Saturation Dependence of Effective Diffusion Coefficient in Unsaturated Argillite Micro-fracture by Lattice Boltzmann Method. Transport in Porous Media, 2017, 117, pp.149-168. 10.1007/s11242-017-0826-z . cea-02421902

HAL Id: cea-02421902

https://hal-cea.archives-ouvertes.fr/cea-02421902

Submitted on 5 Jan 2021

HAL is a multi-disciplinary open access archive for the deposit and dissemination of scientific research documents, whether they are published or not. The documents may come from teaching and research institutions in France or abroad, or from public or private research centers.
L'archive ouverte pluridisciplinaire HAL, est destinée au dépôt et à la diffusion de documents scientifiques de niveau recherche, publiés ou non, émanant des établissements d'enseignement et de recherche français ou étrangers, des laboratoires publics ou privés.

$$
\text { Copyright }
$$




\title{
Computation of saturation dependence of effective diffusion coefficient in unsaturated argillite micro-fracture by lattice Boltzmann method
}

\author{
Alain Genty · Soukaina Gueddani · Magdalena \\ Dymitrowska
}

Received: date / Accepted: date

\begin{abstract}
In the context of radioactive waste repository in deep argillaceous geological formations studies, the effective diffusion coefficient inside a micro-fracture was computed as a function of its saturation level. The micrometric fracture geometry was extracted from the host-rock excavated damaged zone surrounding the repository. It was obtained from X-ray $\mu$-tomography of an Opalinus clay sample from the Mont-Terri laboratory with a $0.7 \mu \mathrm{m}$ voxel resolution. The computations were performed using two Two-Relaxation Time lattice Boltzmann models. The first one, a phase separation model, was used to extract the connected liquid structure inside the fracture for a given saturation. The second one, a diffusion model, was used to compute diffusion in the liquid and to calculate the effective diffusion coefficient for the associated saturation. The calculated curve depicting the effective diffusion dependence on saturation was found to be quasi-linear and to qualitatively match a Maxwell expression for saturations lower than 0.8 .
\end{abstract}

Keywords Lattice Boltzmann method · Effective diffusion · Clay · Unsaturated · Fracture

\section{Introduction}

In the context of high-level long-lived radioactive waste management, deep geological repository is considered as a potential solution by several countries. In France, the geological layer of interest is the Callovo-Oxfordian indurated argillite (Delay et al., 2007) as in Switzerland, the Opalinus Clay, an Aalenian shale is of concern (Marschall et al., 2005). Those Jurassic clays were selected as potential host-rocks mainly because of their low permeability and

A. Genty

Den-Service de thermo-hydraulique et de mécanique des fluides (STMF), CEA, Université Paris-Saclay, F91191 Gif sur Yvette, France

Tel.: $+33-1-69088357$

Fax: $+33-1-69085242$

E-mail: alain.genty@cea.fr

S. Gueddani

Institut de Radioprotection et de Sûreté Nucléaire, IRSN, LETIS, F-92262, Fontenay-aux-Roses, France

M. Dymitrowska

Institut de Radioprotection et de Sûreté Nucléaire, IRSN, LETIS, F-92262, Fontenay-aux-Roses, France 
their high radionuclides retention capacity and a large set of works were devoted to their properties and behavior characterization over the last thirty years.

In the framework of geological repository performance assessment, the migration of radionuclides from the waste towards the biosphere and the identification of preferential pathway is a key point. The building of a repository inside indurated clays will induce the formation of an Excavation Damaged Zone (EDZ) around the drifts, galleries and vaults. Inside the EDZ, fractures of different size are created inside the clay matrix due to hydromechanical decompression (Matray et al., 2007). The EDZ and especially the fracture network may form a preferential diffusion pathway for some radionuclides as, in particular, the ones that hardly diffuse inside the clay matrix like big radius anions (Dagnelie et al., 2015) or those incorporated into colloidal particles (Alonso et al., 2011).

The saturation state of the EDZ fractures is expected to vary during the repository life from almost unsaturated state during the construction and the operation phase due to desaturation process of the geological media in contact with ventilation air (Matray et al., 2007) to a resaturated state reached several tens of thousand years after the repository closure (Poller et al., 2011). In between, anaerobic corrosion of the waste canisters' steel is expected to produce hydrogen (Xu et al., 2008) which may induce two-phase flow inside EDZ (Croisé et al., 2011; Poller et al., 2011) and decrease the fractures' saturation level.

Radionuclide transport simulations performed in the repository performance assessment context then need to take into account the saturation state of the EDZ fractures. As the main transport process expected to occur in very low permeable media such as argillaceous media of interest is diffusion (Rübel et al., 2002; Patriarche et al., 2004), the saturation state of the porous media is accounted for inside the used macroscopic numerical models through the use of an effective diffusion coefficient depending on the saturation level of the geological media.

The experimental determination of the effective diffusion coefficient as a function of saturation is a difficult task and results in dispersed values (Savoye et al., 2010). Numerical approach thus appeared as an interesting complementary tool. In this work, we used two lattice Boltzmann models to perform the computations. The first one, a Two-Relaxation Time phase separation model allowing to access liquid phase geometry for different saturation state was described in sections 3 and 3.1. The second one, a Two-Relaxation Time diffusion model that allows to calculate the effective diffusion coefficient for a given saturation is described in sections 3 and 3.2. We then focus on the geometry of one EDZ fracture with micrometric aperture obtained from $\mu$-tomography imaging described in section 4 . The results obtained are presented in section 5 and 5.1 and discussed in section 6 .

\section{Effective diffusion in unsaturated porous media}

Macroscopic diffusive transport process in unsaturated porous media is described by the diffusion equation (1) (Marsily, 1986; Bear and Bachmat, 1991) derived from the combination of the balance equation and Fick's law for the solute

$$
\frac{\partial \theta C}{\partial t}=\operatorname{div}\left(D_{e}(\theta) \nabla C\right)
$$

where $\theta\left[\mathrm{m}^{3} \cdot \mathrm{m}^{-3}\right]$ is the water content, $C\left[\mathrm{~mol} \cdot \mathrm{m}^{-3}\right]$ is the solute concentration, $t[\mathrm{~s}]$ is time and $D_{e}\left[m^{2} \cdot s^{-1}\right]$ is the solute effective diffusion coefficient.

In practice, the effective diffusion coefficient $D_{e}$ is found to be lower than the molecular diffusion coefficient $D_{0}\left[m^{2} \cdot s^{-1}\right]$ and a large amount of works have aimed at linking the 
$D_{e} / D_{0}$ ratio to porosity and saturation (or water content) (van Brakel and Heertjes, 1974; Epstein, 1989; Boudreau, 1996; Saripalli et al., 2002; Hu and Wang, 2003; Shen and Chen, 2007; Ghanbarian et al., 2013). Following van Brakel and Heertjes (1974), the effective diffusion coefficient can be written as (2)

$$
D_{e}=\theta D_{p}=\theta \frac{\delta}{\tau^{2}} D_{0}
$$

where $D_{p}\left[m^{2} \cdot s^{-1}\right]$ is the pore diffusion coefficient, and geometric factors $\delta[-]$ and $\tau$ $[-]$ are the constrictivity factor and the diffusive tortuosity respectively (Voutilainen et al., 2013).

The diffusive tortuosity factor accounts for the fact that the diffusive pathway along a given interval is larger in a porous medium than in free water due to the presence of the solid. The $\tau$ value is then larger than one and increases as the diffusive pathway length increases. The constrictivity factor accounts for the fact that along a given diffusive pathway the diffusion cross-section can vary and that diffusion is limited by the narrowest throat sections. The $\delta$ value is then lower than one and decreases as the ratio of the smallest throat cross-section to the mean cross-section decreases.

Numerous models for $D_{e}$ dependence on porosity and saturation are available in literature (see van Brakel and Heertjes (1974); Hu and Wang (2003); Moldrup et al. (2004); Shen and Chen (2007); Ghanbarian et al. (2013) for reviews) and the most common used one is the Archie's law (Archie, 1942) given by (3)

$$
D_{e}=S^{n} \omega^{m} D_{0}
$$

where $\omega[-]$ is the porosity, $S[-]$ is the saturation defined as $\theta / \omega, n$ and $m$ are parameters. Note that $m$ (the "cementation exponent" linked to porosity) and $n$ (the "saturation exponent") are found to vary from 1 to 3 in literature with an average value around 2 for real porous media (See Hamamoto et al. (2010) for a review). It is to mention that theoretical works on effective diffusion dependence on saturation are still currently of interest (Ghanbarian et al., 2015; Yang et al., 2016).

In this work, we used TRT Lattice Boltzmann models to compute effective diffusion coefficient $D_{e}(S)$ as a function of saturation for a fracture geometry extracted from the excavated damaged zone of an indurated clay following an identical procedure as the one described in Chau et al. (2005), Zhang et al. (2012) and Genty and Pot (2014).

\section{TRT lattice Boltzmann Models for two-phase distribution and diffusion computations}

We choose to use a Two Relaxation Time lattice Boltzmann model (Ginzburg, 2005; Ginzburg et al., 2008; Humière and Ginzburg, 2009) to simulate the liquid and gas phases distribution inside our fracture geometry and to compute the diffusion of a non-reactive solute in the liquid. The properties of the used lattice Boltzmann models are described as synthetically as possible hereafter and the reader can refer to Genty and Pot $(2013,2014)$ for more details.

The lattice Boltzmann models mimic the microscopic movement of discrete fluid particles through an evolution equation describing local collisions of distributions of populations, $\left\{f_{q}\right\}$, at the nodes, $\mathbf{r}$, of a fixed lattice, and further propagation of the populations to neighbour nodes, according to their unit microscopic velocity vector, $\left\{\mathbf{c}_{q}\right\}$. The microscopic velocity vectors set is composed of the $Q$ vectors $\left\{\mathbf{c}_{q}\right\}=\left\{c_{q \alpha}, \alpha=1, \ldots, d, q=0, \ldots, Q-1\right\}$, 
where $d$ is the space dimension of the lattice. In the TRT scheme, link-basis vectors are constructed for each pair of opposite velocities, $\mathbf{c}_{q}$ and $\mathbf{c}_{\bar{q}}\left(\mathbf{c}_{\bar{q}}=-\mathbf{c}_{q}\right)$ so that the collision operator can be divided into its symmetric (even) and anti-symmetric (odd) parts (Ginzburg, 2005). The TRT evolution equation of the fluid then writes (Ginzburg et al., 2008):

$$
\begin{aligned}
& f_{q}\left(\mathbf{r}+\mathbf{c}_{q}, t+1\right)=f_{q}(\mathbf{r}, t)+\lambda_{e} n_{q}^{+}+\lambda_{o} n_{q}^{-}+S_{q}^{-}, q=0, \cdots, Q-1, \\
& n_{q}^{ \pm}=f_{q}^{ \pm}-e_{q}^{ \pm}, f_{q}=f_{q}^{+}+f_{q}^{-}, f_{q}^{ \pm}=\frac{1}{2}\left(f_{q} \pm f_{\bar{q}}\right), \mathbf{c}_{0}=\mathbf{0}, f_{0}=f_{0}^{+}, f_{0}^{-}=0 \\
& S_{q}^{-}=t_{q}^{*} \sum_{k=1}^{k=N}\left(\mathbf{c}_{q} \cdot \mathbf{F}_{k}\right), k=1, \ldots, N
\end{aligned}
$$

where $\left\{e_{q}^{ \pm}\right\}$are prescribed equilibrium functions, $\left\{S_{q}^{-}\right\}$is a sink or source term, $t_{q}^{*}$ are the isotropic weights of the population distribution, and $\mathbf{F}_{k}$ are force components.

Stability conditions of the model impose that the eigenvalue functions $\Lambda_{e}$ and $\Lambda_{o}$ of the two eigenvalues of the TRT collision operator, $\lambda_{e}$ and $\lambda_{o}$ are positive (Ginzburg et al., 2008):

$$
\begin{aligned}
& \Lambda_{e}=-\left(\frac{1}{2}+\frac{1}{\lambda_{e}}\right), \Lambda_{o}=-\left(\frac{1}{2}+\frac{1}{\lambda_{o}}\right) \\
& \Lambda_{e o}=\Lambda_{e} \Lambda_{o},-2<\lambda_{e}, \lambda_{o}<0
\end{aligned}
$$

The following equilibrium functions, $e_{q}^{ \pm}$, were used to model the Stokes and diffusion equations (Ginzburg et al., 2008):

$$
\begin{aligned}
& e_{0}^{+}=e_{0}=\rho-\sum_{q=1}^{Q-1} e_{q}^{+}, e_{q}^{+}=t_{q}^{*} c_{s}^{2} \rho, q=1, \cdots, Q-1, \\
& e_{q}^{-}=t_{q}^{*} \mathbf{J} \cdot \mathbf{c}_{q}
\end{aligned}
$$

where $\rho$ and $\mathbf{J}$ are the microscopic mass and momentum quantities defined from the local populations $f_{q}$ and calculated at each node $\mathbf{r}$ according to:

$$
\rho=\sum_{q=0}^{Q-1} f_{q}, \mathbf{J}=\rho \mathbf{u}=\sum_{q=0}^{Q-1} f_{q} \mathbf{c}_{q}
$$

where $\mathbf{u}$ is the macroscopic velocity and $c_{s}=1 / 3$ the sound velocity.

\subsection{Two-phase TRT lattice Boltzmann model}

On the basis of the generic lattice Boltzmann model described in section 3, the liquid-gas phases distributions were simulated using the $3 \mathrm{D}$ nineteen-velocities model (D3Q19) where the weights are $t_{I}^{*}=1 / 6, t_{I I}^{*}=1 / 12$, with the Roman numbers equal to $\left\|\mathbf{c}_{q}\right\|^{2}$ and $\lambda_{e}$ is calculated from the kinematic viscosity of the liquid $v=(1 / 3) \Lambda_{e}$ for the case of incompressible Stokes flow.

Following Genty and Pot (2013) we arbitrarily set $v=1 / 6[l . u .]^{2} \cdot[t . u .]^{-1}$ and we used $\Lambda_{e o}=3 / 16$ as this value gives exact Poiseuille profile between parallel plates (Ginzburg and d'Humières, 2003). The $\lambda_{o}$ value was then calculated from equation (5). We used two force components in our model: $\mathbf{F}_{G}$ to simulate the attractive fluid-fluid short-range interactions that describe surface tension effects and $\mathbf{F}_{W}$ the attractive or repulsive fluid-solid interactions that simulate hydrophilic of hydrophobic surfaces. 
The fluid-fluid attractive interaction force, $\mathbf{F}_{G}$, was defined according to Shan-Chen approach (Shan and Chen, 1993, 1994) along:

$$
\mathbf{F}_{G}=-\psi(\mathbf{r}, t) \sum_{q} t_{G q}^{*} G \psi\left(\mathbf{r}+\mathbf{c}_{q}, t\right) \mathbf{c}_{q}
$$

where $G<0$ is the parameter that controls the attractive interaction strength, $\psi$ is a potential function that depends on the macroscopic density, $\rho$, and can be varied arbitrarily, with $\psi(\rho)=1-\exp (-\rho(\mathbf{r}, t))$ (Raiskinmäki et al., 2000) and the weights $t_{G q}^{*}$ are $t_{G, 0}^{*}=0, t_{G, I}^{*}=$ $2, t_{G, I}^{*}=1$ (Raiskinmäki et al., 2000).

The solid-fluid interaction force, $\mathbf{F}_{W}$, was defined according to Martys and Chen (1996) and Raiskinmäki et al. (2000):

$$
\mathbf{F}_{W}=-\psi(\mathbf{r}, t) \sum_{q} t_{W q}^{*} W s\left(\mathbf{r}+\mathbf{c}_{q}, t\right) \mathbf{c}_{q}
$$

where $s=0$ or 1 if the site $\mathbf{r}$ is fluid or solid, respectively, $W$ is the parameter that controls the hydrophobicity of the solid surface and the weights $t_{W q}^{*}$ are set to $t_{W, 0} *=0, t_{W I}^{*}=2$, $t_{W, I I}^{*}=1$ (Raiskinmäki et al., 2000).

The resulting numerical model was extensively tested in Genty and Pot (2013) and was found to mimic the liquid-gas phase separation process of a binary fluid. The modeled phase separation process appears to be similar to a physical spinodal decomposition process (Appert and Zaleski, 1990) where final steady state is controlled by surface tension forces. The described model was successfully applied to the simulation of real air-water distribution in soil pores (Pot et al., 2015).

\subsection{Diffusion TRT lattice Boltzmann model}

On the basis of the generic lattice Boltzmann model described in section 3, diffusion of a non-reactive solute in the liquid phase was simulated using the $3 \mathrm{D}$ seven-velocities model (D3Q7) where the weights are $t_{I}^{*}=1 / 2$ and $\lambda_{0}$ is set according to the molecular diffusion coefficient of the solute in liquid phase $D_{0}=-c_{s}^{2}\left(\frac{1}{2}+\frac{1}{\lambda_{o}}\right)$. Following Pot et al. (2010) we used $D_{0}=0.5[l . u .]^{2} \cdot[t . u .]^{-1}$. We fixed $\Lambda_{e o}=1 / 32$ and calculated $\lambda_{e}$ from equation (5). As the solute is non reactive, the source term $S_{q}=0$. Note that in the case of diffusion only, the macroscopic velocity is set to zero, so that $\mathbf{J}=0$.

\section{Fracture description}

The micro-fracture used in this work originated from the Opalinus Clay of the Mont Terri Rock laboratory in Switzerland. The fracture was chosen among the available fracture geometries obtained from X-ray $\mu$-tomography of clay samples collected inside the EDZ surrounding the borehole of the Ventilation Experiment II (Mayor et al., 2007).

\subsection{General description}

The selected fracture was observed inside a cubic-millimeter volume of a clay sample. It was a mainly planar sub-horizontal fracture with a mean aperture of about $6 \mu \mathrm{m}$. The initial 
image of the fracture was obtained from high resolution X-ray tomography performed with the radiation provided by the European Source of Synchrotron radiation and the techniques of the ID 19 line dedicated to micro-tomography (ESRF Grenoble, France). The Synchrotron operates with a high current and a very intense flow of photons that allows as short as possible acquisitions. The use of a FReLon camera CCD 14 bits developed specifically and suitable optics makes it possible to obtain sizes of pixels from 40 to $0.4 \mu \mathrm{m}$. The resolution reached for our sample was $0.7 \mu \mathrm{m}$ with a total dimension of about $1000^{3}$ voxels. The 3D picture was constituted of voxels of different grey levels characterizing the X-ray adsorption coefficient of the voxel material. The fracture was then distinguished from the clay matrix using a thresholding operation.

\subsection{Geometry for LBM computations}

From the initial 3D $\mu$-tomography picture, we extracted a sub-part of the fracture and mirrored it in the $x$ direction. The fracture geometry used for our LBM computations was then included in a $100 \times 152 \times 18[l u]^{3}$ LBM network presented in Figure 1 . Note that the $z$ di-
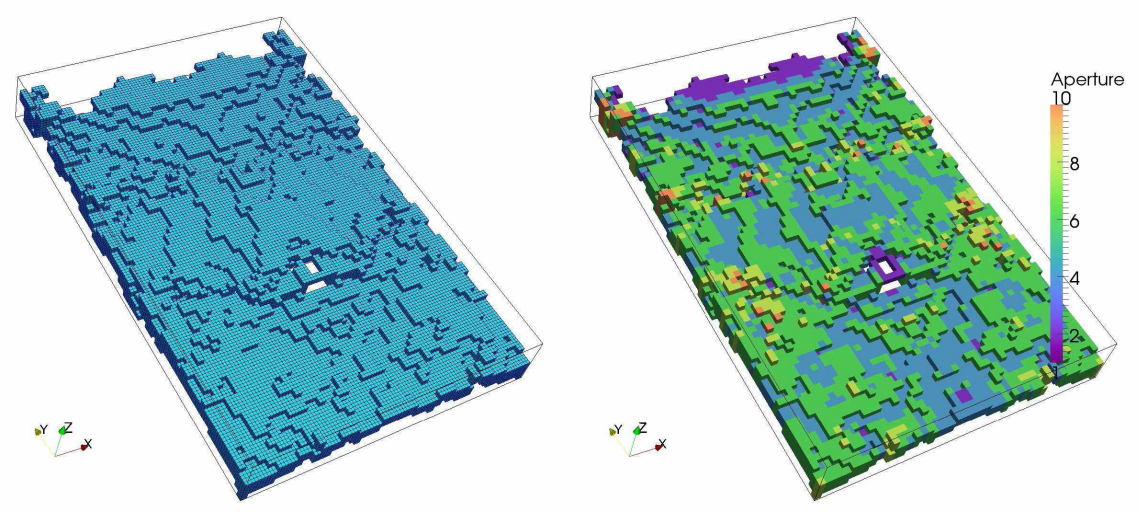

Fig. 1 Fracture geometry with voxels representation (left) and local aperture in $\mu m$ (right).

rection was reduced as much as possible in order to remove a large volume of clay matrix where flow and diffusion are not considered. The fracture was mirrored in the $x$ direction that we choose as main diffusion direction computation for LBM symmetry purpose. The porosity of the selected sample volume was $\omega=0.256$.

\section{Saturation calculation}

The saturation computations were performed on the fracture geometry presented in Figure 1 using the lattice Boltzmann phase separation model briefly described in section 3.1. The main model parameters values used to perform the phase separation computations were $G=-0.16$ and $W=0.15$ (we considered here fully wetting walls) (Genty and Pot, 2014). At initial time, a density $\rho_{\text {init }}$ was imposed inside the void space of the fracture. The presence 
of the wetting walls induced spontaneous phase separation leading to a low density phase (gas) and a higher density phase (water). The computed saturation $S$ in the void space was expected to follow the analytical expression $S=\left(\rho_{\text {init }}-\rho_{\text {gas }}^{t h}\right) /\left(\rho_{\text {liq }}^{t h}-\rho_{\text {gas }}^{t h}\right)$ deduced from the phase diagram described in Genty and $\operatorname{Pot}$ (2013) (for $G=-0.16$, theoretical gas and liquid densities are $\rho_{\text {gas }}^{\text {th }}=0.03$ and $\rho_{\text {liq }}^{\text {th }}=2.42$ respectively) and from mass conservation (the total mass of the initial fluid of density $\rho_{\text {init }}$ must be equal to the sum of the mass of the low density phase and the high density phase). We noticed that after steady state was reached the maximum and minimum calculated density values were slightly different than the theoretical ones and that phase separation did not oceur for initial densities close to the phase diagram boundary densities. But it is to note that the theoretical phase diagram described in Genty and Pot (2013) is valid without walls and with an initial density $\rho_{\text {init }}=\ln 2$ only. We also noticed that small negative densities that we attributed to gas phase were obtained at few lattice nodes.

Density values $\rho_{\text {init }}$ were selected in the range $\simeq 0.6-1.8$ to perform the phase separation. For some representative saturations $S$, the distribution of calculated gas bubbles inside the fracture are presented in Figure 2.

On the basis of the calculated liquid-gas distributions inside the pore space, a basic thresholding algorithm allowed to extract the liquid phase and the gas phase. The density threshold value was set to $\left(\rho_{\text {liq }}^{\text {cal }}+\rho_{\text {gas }}^{\text {cal }}\right) / 2 \simeq \rho_{\text {liq }}^{\text {cal }} / 2$ where higher density was considered as liquid and lower density as gas. For the diffusion computations, we only took into account diffusion in the liquid phase. Note that because the resulting thresholded liquid phase was not necessary connected, an additional liquid cluster removal algorithm was applied in order to extract the connected liquid phase. In the context of the $D 3 Q 7$ lattice used for the diffusion computations, only connections through voxels faces were considered.

A summary of the connected liquid phase extraction procedure is pictured for $S=0.48$ in Figure 3. We therefore conducted effective diffusion calculation for the liquid phase geometries that corresponded to a given final saturation. Note that because of the application of the cluster removal algorithm, final saturations can differ from the ones initially calculated. For all the selected initial density values $\rho_{\text {init }}$, the initial calculated saturation $(S)$ and the ones calculated after cluster removal algorithm $\left(S_{c}\right)$ are summarized in Table 1.

Table 1 calculated saturations for selected initial density

\begin{tabular}{cccccccccccc}
\hline$\rho_{\text {init }}$ & 0.60 & 0.70 & 0.72 & 0.73 & 0.75 & 0.77 & 0.78 & 0.80 & 0.90 & 1.00 & 1.10 \\
\hline$S$ & 0.20 & 0.25 & 0.26 & 0.27 & 0.28 & 0.29 & 0.30 & 0.31 & 0.37 & 0.42 & 0.48 \\
\hline$S_{c}$ & 0.00 & 0.06 & 0.07 & 0.07 & 0.07 & 0.09 & 0.19 & 0.24 & 0.30 & 0.39 & 0.45 \\
\hline \hline$\rho_{\text {init }}$ & 1.20 & 1.30 & 1.40 & 1.50 & 1.60 & 1.65 & 1.70 & 1.71 & 1.72 & 1.73 & 1.75 \\
\hline$S$ & 0.54 & 0.59 & 0.65 & 0.71 & 0.77 & 0.79 & 0.84 & 0.86 & 0.88 & 0.94 & 1.00 \\
\hline$S_{c}$ & 0.52 & 0.58 & 0.64 & 0.70 & 0.76 & 0.78 & 0.83 & 0.85 & 0.87 & 0.94 & 1.00 \\
\hline
\end{tabular}

\subsection{Effective diffusion calculation}

For a given liquid phase geometry extracted according to the procedure described in the previous section, we computed a tracer diffusion using the diffusion TRT lattice Boltzmann 


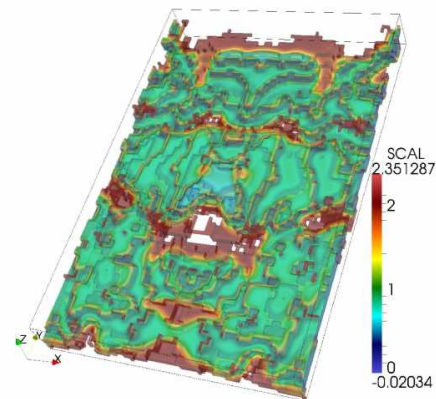

(a) $S=0.25$

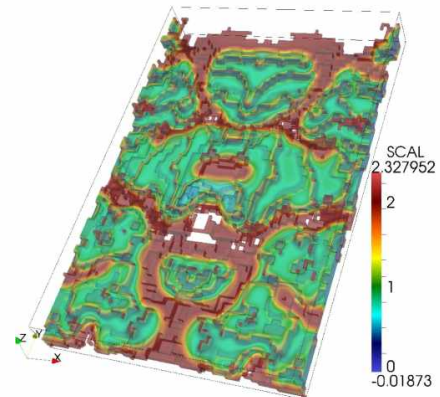

(c) $S=0.37$

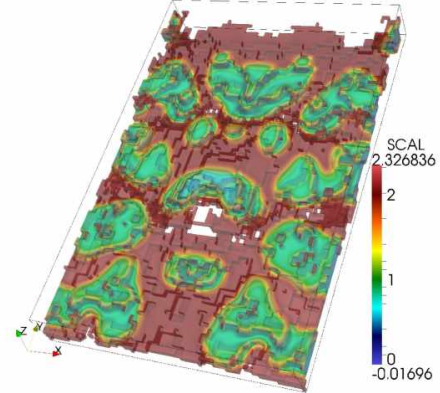

(e) $S=0.54$

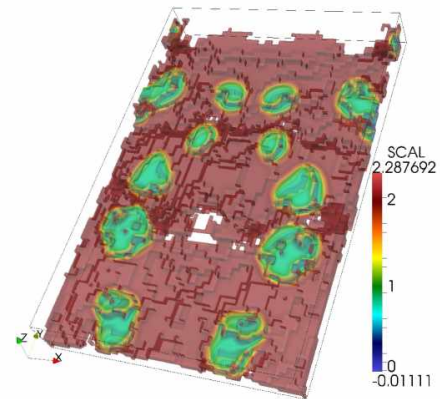

(g) $S=0.79$

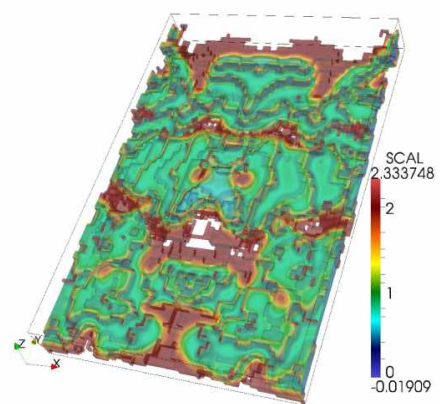

(b) $S=0.30$

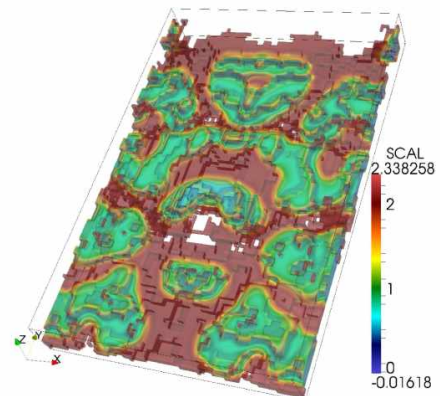

(d) $S=0.48$

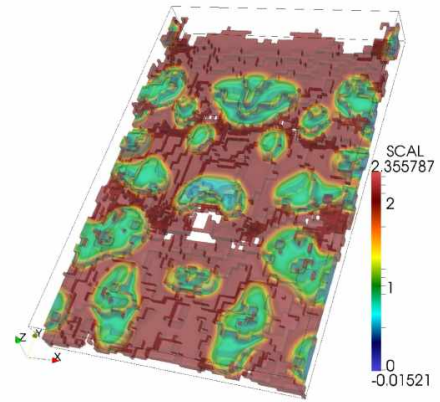

(f) $S=0.65$

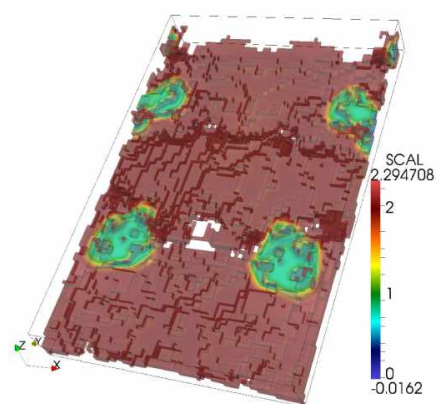

(h) $S=0.88$

Fig. 2 3D views of the calculated gas bubble (in green) and corresponding related saturations for several initial densities; the solid phase has been removed. 

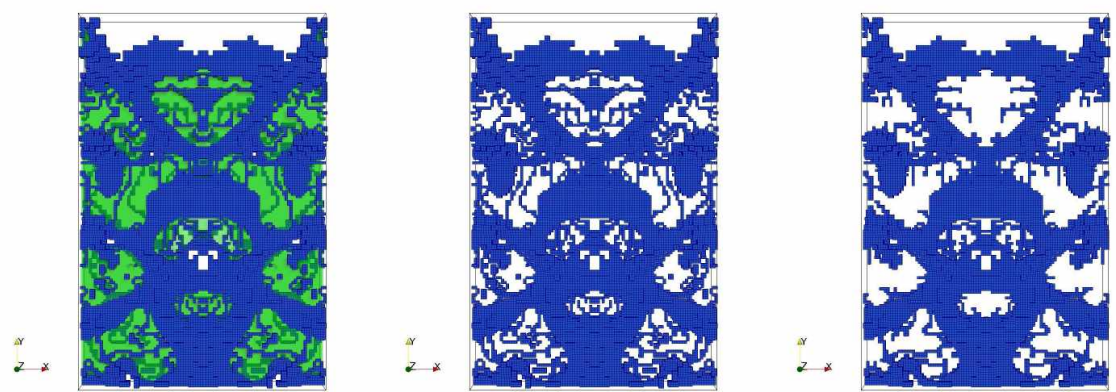

Fig. 3 Summary of the liquid phase extraction procedure for $S=0.48$. Thresholded liquid phase in blue with gas bubbles in green (left). Thresholded liquid phase only (center). Thresholded liquid phase after cluster removal (only connections through voxel faces are considered) (right).

model briefly described in section 3.2. At initial time, we set a tracer concentration $C_{i}=1$ inside the liquid in the symmetry plane $x=x_{0}=50[l . u$. $]$ and a zero concentration everywhere else. Due to symmetry reasons, diffusion was expected to take place perpendicularly to the initial concentration plane and along the $x$ axis. Considering $1 \mathrm{D}$ diffusion along $x$ axis for an infinite medium with the particular Dirac initial tracer concentration $C_{i}=1$ for $x=x_{0}$, the tracer concentration along $x$ is expected to match the classical analytical expression (10) for the diffusion equation (1)

$$
C(x, t)=\frac{C_{0}}{\sqrt{4 \pi D_{p} t}} \exp -\frac{\left(x-x_{0}\right)^{2}}{4 D_{p} t}
$$

where $C_{0}$ is the initial solute concentration defined through the initial tracer mass $M_{0}$ by $M_{0}=C_{0} \phi_{x_{0}} L_{y} L_{z}$ with $\phi_{x_{0}}$ the porosity of plane $x=x_{0}$ (defined as the number of liquid sites over the total number of sites of the plane $x=x 0=50[l . u].), L_{y}=152[l . u$. $]$ and $L_{z}=18[l . u$. $]$.

We first computed diffusion for the fully saturated micro-fracture $(S=1)$ (Cf. Figure 1) over a computation time $t=300[t . u$. $]$ large enough for the concentration plume to diffuse through a major part of the micro-fracture volume but small enough to keep a zero concentration on the edges $x=0[l . u$. $]$ and $x=100[l . u$. $]$ to guarantee the applicability of the analytical expression (10) valid for an infinite medium. The computed concentration plume inside the liquid at $t=300[t . u$. $]$ is presented in Figure 4.

For each $x$ plane value of our LB network, we calculated the mean concentration as $C=$ $\left(\sum_{y, z} C(x, y, z)\right) / \phi_{x} L_{y} L_{z}$ and the resulting concentration profile along $x$ is shown in Figure 5.

The pore diffusion coefficient $D_{p}$ for the saturated micro-fracture was calculated by fitting the LBM calculated concentration profile with the analytical expression (10), where $D_{p}$ was varied, through a minimum $L_{2}$ error objective. The analytical fit presented in Figure 5 was obtained with a value of $D_{p}=0.396[l . u .]^{2} \cdot[t . u .]^{-1}$, that is to say $D_{e}=\theta D_{p}=0.256 \times$ $0.396=0.102[l . u .]^{2} \cdot[t . u .]^{-1}$. Note that according to expression (3), exponent $m$ is then equal to $m=1.2$ which is close to the value of 1.3 found for unconsolidated sands by Archie (1942) and for glass spheres by Friedman (2005).

An identical computation procedure was applied for all the extracted liquid geometries corresponding to micro-fracture saturations given in Table 1. Some computed concentration plumes at $t=300[t . u$. $]$ are presented in Figure 6 for relevant saturation values. 


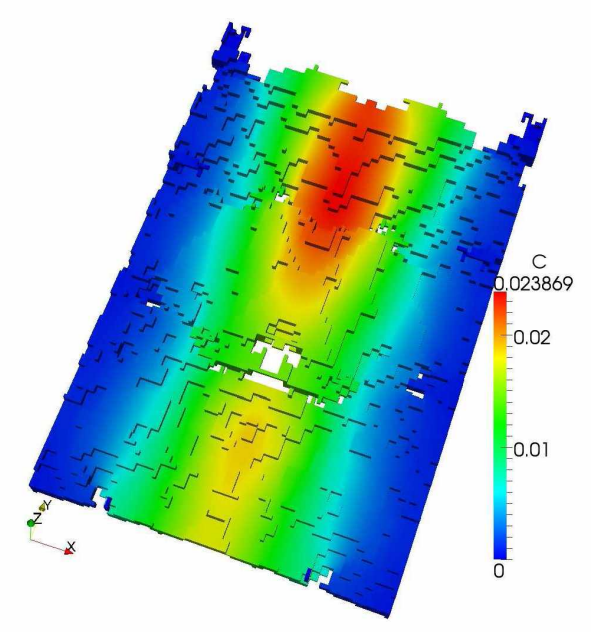

Fig. 4 3D view of the tracer concentration plume inside the saturated $(S=1)$ micro-fracture at $t=300[t . u$. $]$.

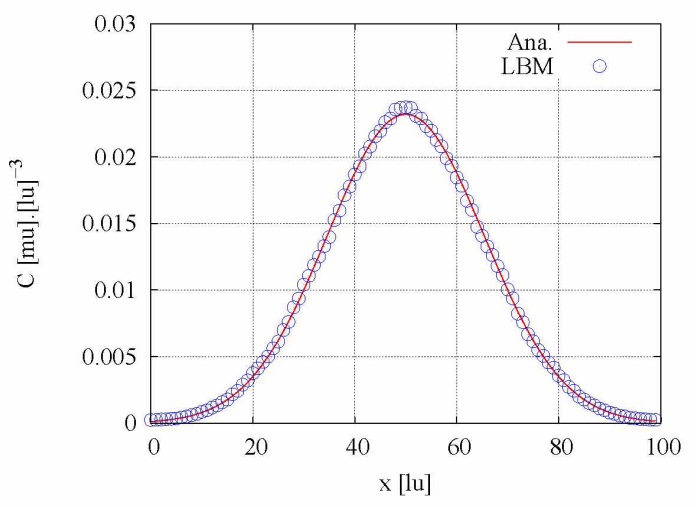

Fig. 5 Concentration profiles along $x$ at $t=300[t . u$. $]$ for $S=1.0$.

Table 2 reports the effective diffusion coefficients calculated for each saturation as well as minimal $L_{2}$ error for the fit.

Concentration profiles and analytical fits are presented in Figure 7 for relevant saturation values (namely $S=0.25, S=0.30, S=0.37, S=0.65, S=0.84$ and $S=0.88$ ).

Except for few values like in $S=0.30$ case, a close correspondence was found between calculated concentration profiles and analytical fits with low $L_{2}$ errors (Cf. Table 2). It is to note that the poor fit obtained for the $S=0.30$ stems from the concentration trapping inside dead-end pore close to the symmetry plane (see Figure 6(b)).

The plot of the normalized $D_{e}(S)$ is presented in Figure 8 .

The trend of the $D_{e}(S)$ curve depicted in Figure 8 is quasi-linear with $D_{e}$ decreasing with decreasing $S$. The linear trend found is different than the quadratic one described in literature for real porous media (See Hamamoto et al. (2010) for a review) but similar to the one found for regular porous media made of equal spheres or grains as a function of saturation (Martys, 1999; Jones et al., 2003; Genty and Pot, 2014) or porosity (Xuan et al., 


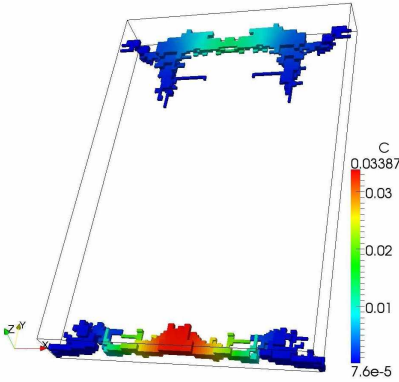

(a) $S=0.25$

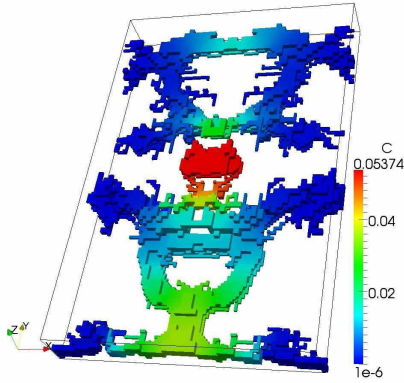

(c) $S=0.37$

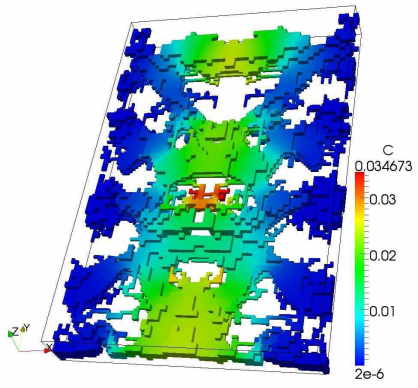

(e) $S=0.54$

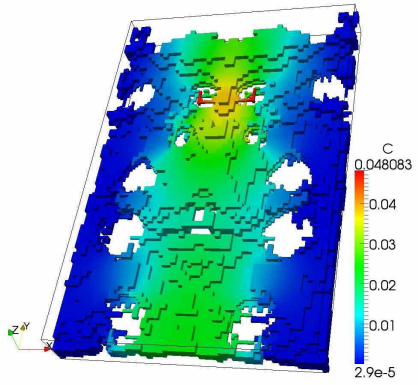

(g) $S=0.79$

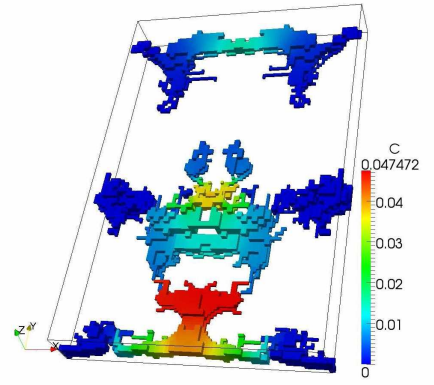

(b) $S=0.30$

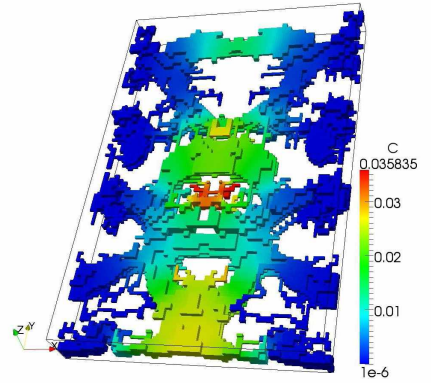

(d) $S=0.48$

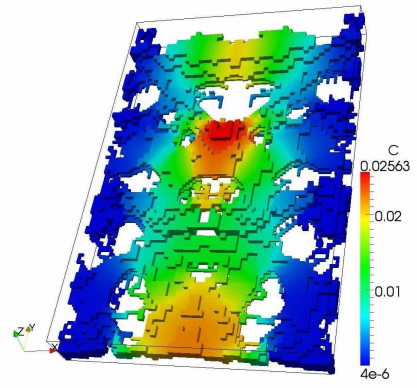

(f) $S=0.65$

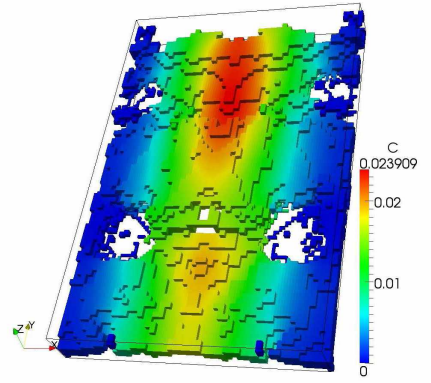

(h) $S=0.88$

Fig. $63 \mathrm{D}$ concentration plume for different saturations at $t=300[t . u$. $]$ 
Table 2 Effective diffusion coefficient

\begin{tabular}{lllll}
\hline$S$ & $\theta$ & $D_{p}$ & $D_{e}$ & $L_{2}$ \\
\hline 1.000 & 0.256 & 0.396 & 0.101 & 0.02 \\
0.939 & 0.240 & 0.414 & 0.099 & 0.02 \\
0.877 & 0.224 & 0.424 & 0.095 & 0.02 \\
0.861 & 0.220 & 0.428 & 0.094 & 0.02 \\
0.836 & 0.214 & 0.438 & 0.094 & 0.02 \\
0.793 & 0.203 & 0.338 & 0.069 & 0.03 \\
0.767 & 0.196 & 0.370 & 0.073 & 0.04 \\
0.709 & 0.181 & 0.353 & 0.064 & 0.05 \\
0.651 & 0.167 & 0.317 & 0.053 & 0.03 \\
0.594 & 0.152 & 0.294 & 0.045 & 0.03 \\
0.540 & 0.138 & 0.285 & 0.039 & 0.03 \\
0.480 & 0.123 & 0.255 & 0.031 & 0.03 \\
0.424 & 0.109 & 0.199 & 0.022 & 0.06 \\
0.367 & 0.094 & 0.167 & 0.016 & 0.07 \\
0.307 & 0.079 & 0.200 & 0.016 & 0.11 \\
0.295 & 0.076 & 0.095 & 0.007 & 0.29 \\
0.289 & 0.074 & 0.220 & 0.016 & 0.08 \\
0.278 & 0.071 & 0.092 & 0.007 & 0.03 \\
0.269 & 0.069 & 0.239 & 0.016 & 0.07 \\
0.263 & 0.067 & 0.082 & 0.005 & 0.33 \\
0.253 & 0.065 & 0.235 & 0.015 & 0.07 \\
0.202 & 0.052 & 0.000 & 0.000 & - \\
\hline
\end{tabular}

2010; Bertei et al., 2013) suggesting that the studied fracture can be considered as regular from a topological point of view with a long-distance correlation structure.

We noticed for saturation $S$ lower than 0.3 an unstable behavior with computations of high and low $D_{e}$ values for very close saturations values near the disconnection one $(\omega=$ 0.20 ). This behavior is suspected to be linked to the coarse refinement level used to describe the fracture geometry. Indeed, for small saturations, a one water voxel difference can results in cluster disconnection or not. This point will be carefully investigated in the next section.

\section{Discussion}

In order to investigate the $D_{e}$ behavior observed for saturation $S$ lower than 0.3 , a new set of computations was conducted on the same fracture geometry but using a 2 times refined network. Each initial voxel of Figure 1 (left) is then divided in $8(0.35 \mu \mathrm{m})^{3}$ voxel leading to a $200 \times 304 \times 36[l u]^{3}$ LBM network. We applied to this refined network the same procedure than the one previously described. For a selected initial density $\rho_{\text {init }}$ we first conducted a phase separation computation and then a diffusion calculation in the connected fluid geometry. We noticed that the new computed gas phase distributions as well as saturation values were not strictly equal to the previous ones but remained close for the saturations and topologically similar for the gas phase distribution as presented in Figure 9. Those small differences were found to have no noticeable impact on extracted connected liquid topology for saturations larger than 0.4 as shown on Figure 10 (c) and (d). This is obviously not the case for saturations lower than 0.4 where despite very similar gas phase distributions for the two discretization level, the remaining connected liquid shape is different (see Figure 10 (a) and (b)). For the initial discretization level (x1), only simple large connected areas are extracted 


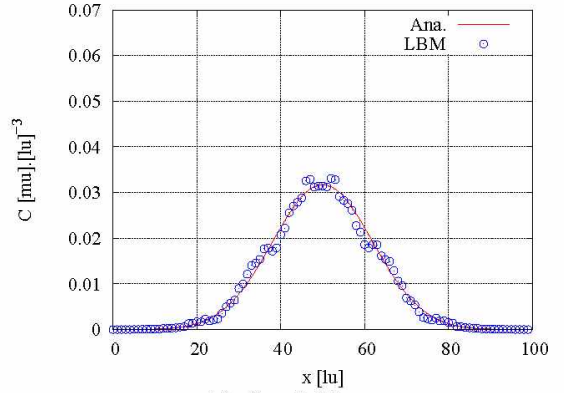

(a) $S=0.25$

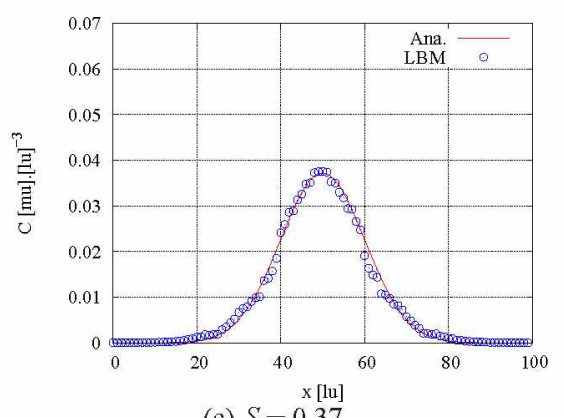

(c) $S=0.37$

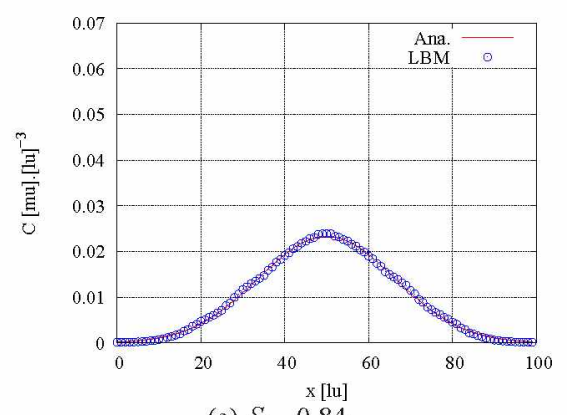

(e) $S=0.84$

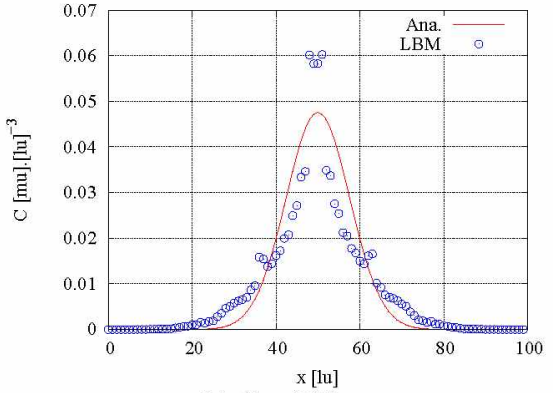

(b) $S=0.30$

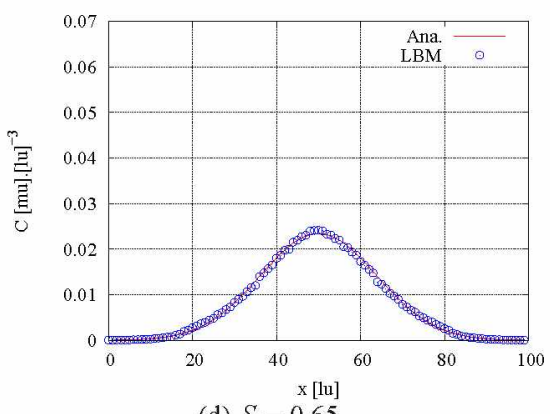

(d) $S=0.65$

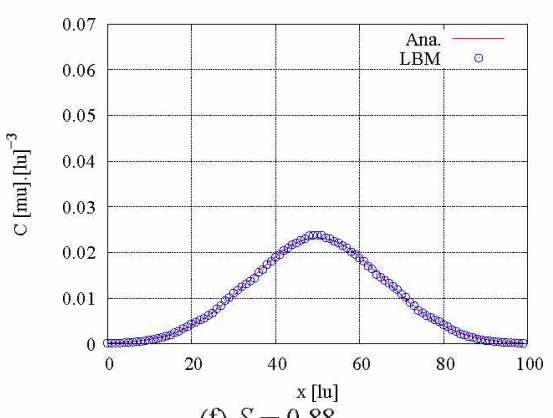

(f) $S=0.88$

Fig. 7 Mean concentration profiles along $x$ for several saturations at $t=300[t . u$. $]$.

(see Figure 10(a)) as for the refined discretization level (x2), individual voxels connections allowed to extract a more complex and more tortuous geometry (see Figure 10(b)).

The numerical results obtained for the refined fracture network are summarized in Table 3 .

The normalized effective diffusion values computed for the two refinement levels are presented in Figure 11.

Figure 11 indicates that the unstable behavior of $D_{e}$ observed for saturation lower than 0.3 disappeared as the lattice refinement increased. This result pointed out that, like for any other mesh based method, lattice Boltzmann model computation accuracy is linked to the lattice refinement. It is to note that in our case, the main part of the computation inaccuracy do not stem from the diffusion calculation part but from the phase distribution 


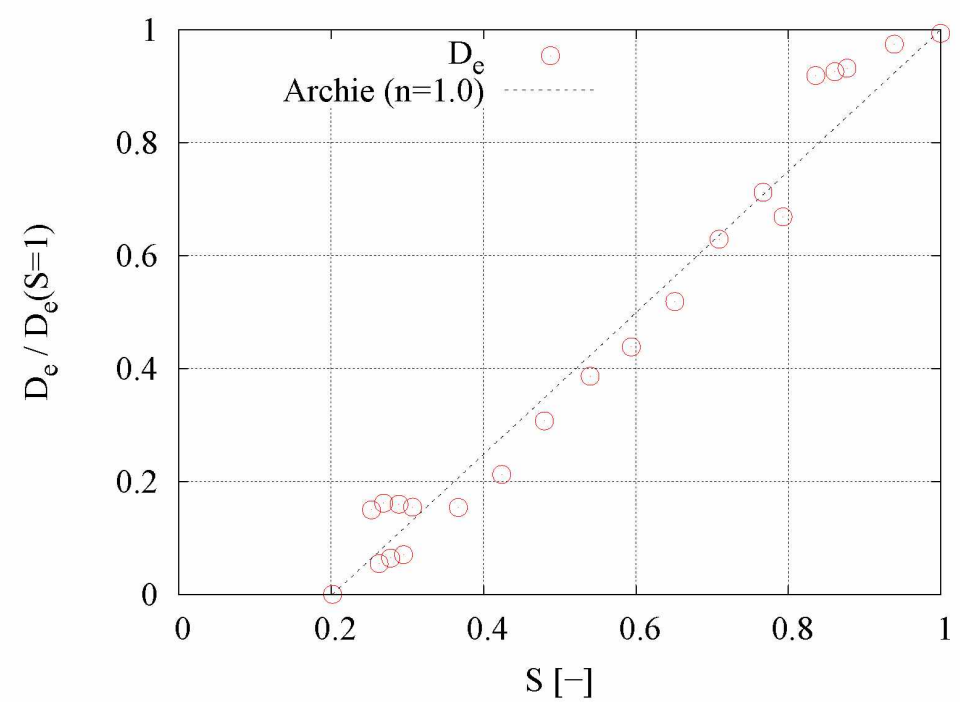

Fig. 8 Effective diffusion coefficient $D_{e}$ as a function of saturation $S$.

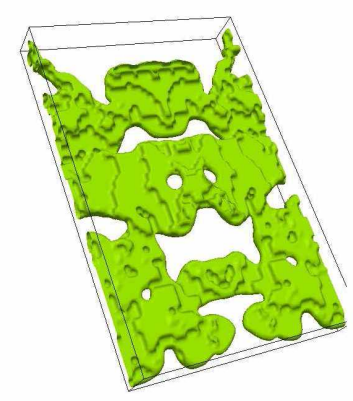

(a) $S \simeq 0.30$, discretization $\mathrm{x} 1$

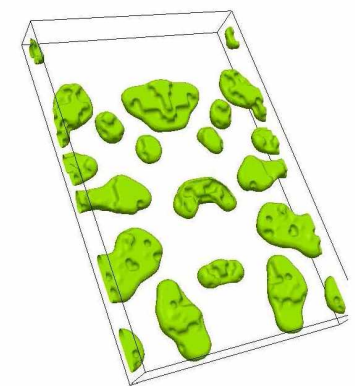

(c) $S \simeq 0.65$, discretization $\mathrm{x} 1$

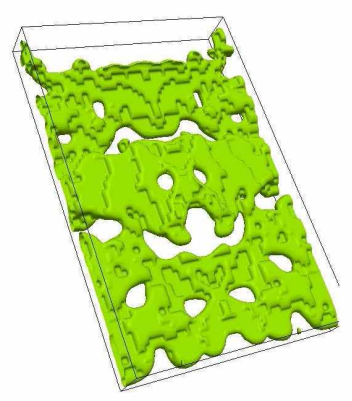

(b) $S \simeq 0.30$, discretization $\times 2$

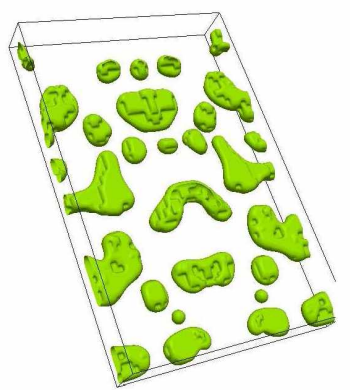

(d) $S \simeq 0.65$, discretization $\times 2$

Fig. 9 Gas distribution (in green) at different discretization levels for $S \simeq 0.30$ and $S \simeq 0.65$. 


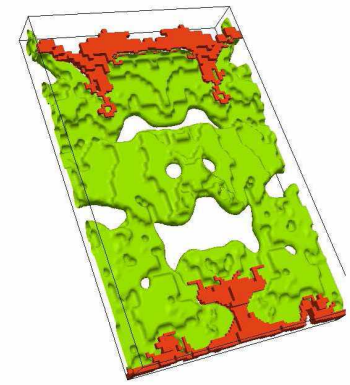

(a) $S \simeq 0.30$, discretization $\mathrm{x} 1$

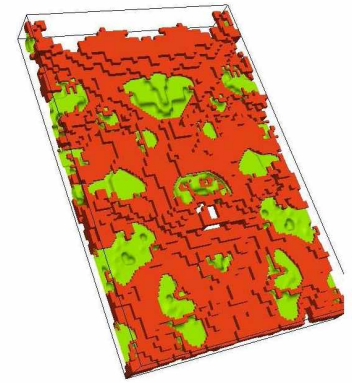

(c) $S \simeq 0.65$, discretization $\mathrm{x} 1$

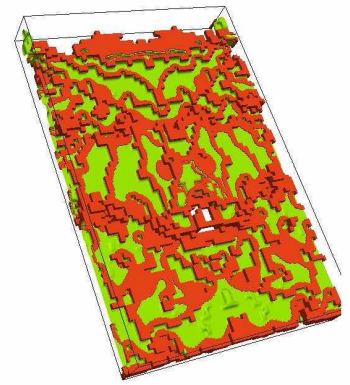

(b) $S \simeq 0.30$, discretization $\mathrm{x} 2$

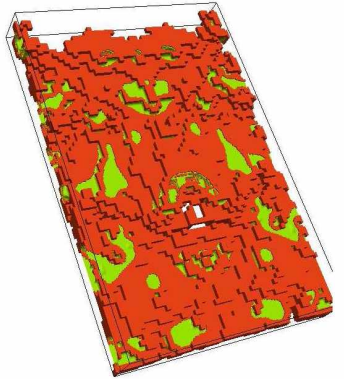

(d) $S \simeq 0.65$, discretization $\mathrm{x} 2$

Fig. 10 Extracted connected liquid topology (in red) at different discretization levels for $S \simeq 0.30$ and $S \simeq$ 0.65 .

Table 3 Effective diffusion coefficient computed for refined geometry

\begin{tabular}{lllllll}
\hline$\rho_{\text {init }}$ & $S$ & $S_{c}$ & $\theta$ & $D_{p}$ & $D_{e}$ & $L_{2}$ \\
\hline 1.75 & 1.00 & 1.00 & 0.256 & 0.398 & 0.102 & 0.02 \\
1.73 & 0.96 & 0.96 & 0.246 & 0.402 & 0.099 & 0.02 \\
1.72 & 0.96 & 0.95 & 0.246 & 0.403 & 0.099 & 0.02 \\
1.71 & 0.81 & 0.81 & 0.208 & 0.428 & 0.089 & 0.02 \\
1.70 & 0.74 & 0.74 & 0.190 & 0.302 & 0.057 & 0.09 \\
1.65 & 0.73 & 0.72 & 0.187 & 0.281 & 0.053 & 0.08 \\
1.60 & 0.70 & 0.70 & 0.179 & 0.319 & 0.057 & 0.05 \\
1.50 & 0.65 & 0.65 & 0.167 & 0.283 & 0.047 & 0.08 \\
1.40 & 0.61 & 0.60 & 0.156 & 0.277 & 0.043 & 0.06 \\
1.30 & 0.56 & 0.55 & 0.144 & 0.216 & 0.031 & 0.08 \\
1.20 & 0.51 & 0.50 & 0.131 & 0.209 & 0.027 & 0.12 \\
1.10 & 0.46 & 0.45 & 0.118 & 0.202 & 0.024 & 0.18 \\
1.00 & 0.41 & 0.40 & 0.105 & 0.196 & 0.021 & 0.16 \\
0.90 & 0.36 & 0.35 & 0.092 & 0.173 & 0.016 & 0.17 \\
0.80 & 0.31 & 0.30 & 0.079 & 0.130 & 0.010 & 0.22 \\
0.78 & 0.30 & 0.29 & 0.077 & 0.127 & 0.010 & 0.22 \\
0.77 & 0.30 & 0.28 & 0.077 & 0.125 & 0.010 & 0.22 \\
0.75 & 0.29 & 0.27 & 0.074 & 0.100 & 0.007 & 0.20 \\
0.73 & 0.28 & 0.26 & 0.072 & 0.093 & 0.007 & 0.20 \\
0.72 & 0.27 & 0.25 & 0.069 & 0.091 & 0.006 & 0.20 \\
0.70 & 0.26 & 0.24 & 0.067 & 0.072 & 0.005 & 0.23 \\
\hline
\end{tabular}




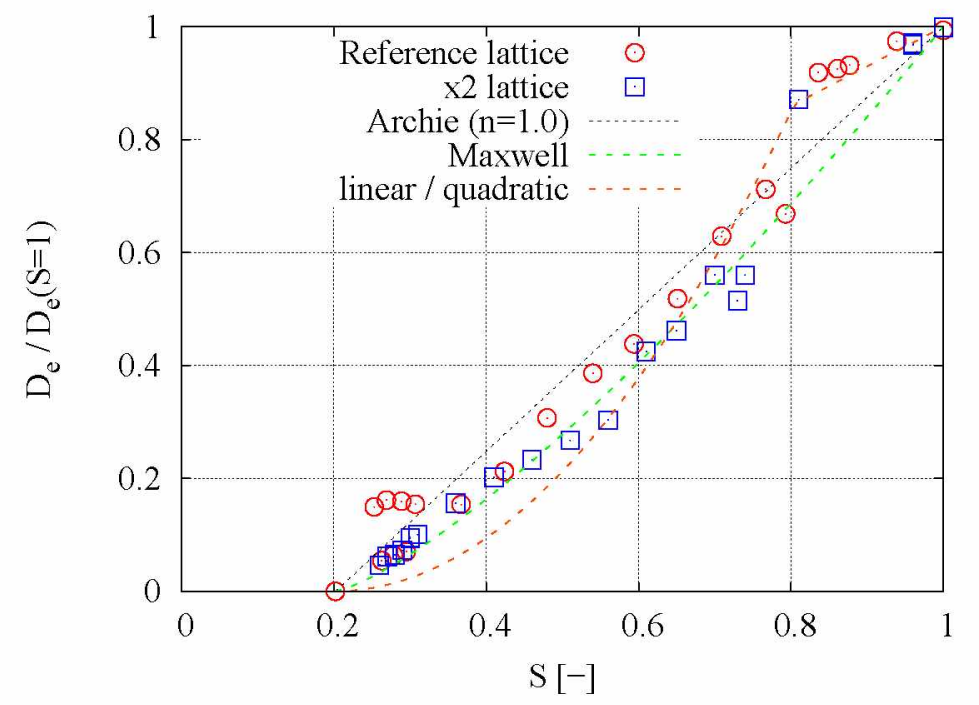

Fig. 11 Normalized effective diffusion coefficient $D_{e}$ as a function of saturation $S$ at two discretisation levels.

computation. Indeed, when pore throats are only represented by few lattice nodes, a one voxel difference in liquid-gas interface position can results in a liquid phase disconnection and the removal of many water paths from the diffusion process. This effect was clearly present with coarse lattice where connected saturation $S_{C}$ were much lower than the initially calculated saturations $S$ (see Table 1 for saturations lower than 0.3 ) but with the refined lattice $S_{C}$ remains within $10 \%$ from $S$ (see Table 3 ).

Figure 11 confirms that the fit of $D_{e}(S)$ using a power law like the one of Archie with an exponent $n=1$ was broadly relevant. Precisely, we plotted an Archie's second law in the form $\left(\left(S-S_{d i s}\right) /\left(1-S_{d i s}\right)\right)^{n}$ as suggested by Balberg (1986) and Martys (1999) with $S_{\text {dis }}=0.2$ the saturation corresponding to the disconnection of the liquid phase. Note that the classical Maxwell equation $D_{e}\left(S_{n}\right)=2 S_{n} / 3-S_{n}$ with $S_{n}=\left(S-S_{\text {dis }}\right) /\left(1-S_{\text {dis }}\right)$ (Maxwell, 1873 ) which is close to the Archie's law using $n=1.3$ (Archie, 1942; Friedman, 2005) gives a close fit of the computed points for saturations lower than 0.8 .

A further analysis of the Figure 11 indicated that the behavior of $D_{e}$ can be divided in two main parts. The first one is quasi linear with a slow increase of $D_{e}$ with $S$ in the range $0.8-1.0$ (points above the Archie line). The second one corresponds to a power law (with an exponent slightly larger than 1 ) in the range $0.2-0.8$ (points below the Archie line). We note that the obtained curve with two parts corresponded to the one proposed by Ghanbarian et al. (2015) using percolation theory and the Effective Medium Approximation with a linear part for saturation greater than 0.8 and a quadratic part for lower saturations. Such a linear/quadratic expression was drawn on Figure 11 for comparison purpose and found to well match the computed points for high saturation values but to disappointingly underestimates the calculated effective diffusion for low saturations.

It is important to emphasize that the results obtained here are valid for diffusion process in this particular fracture geometry only. In the real EDZ argillite media, matrix diffusion is also of concern and resulting effective diffusion calculation in fracture-matrix system deserve additional work using homogenization and up-scaling techniques. Another limitation 
of the presented results consists in the impossibility for the lattice Boltzmann model to take into account water-films thinner than one voxel $(0.35 \mu \mathrm{m}$ for the finest lattice used in this study). A difference between the effective diffusion coefficient calculated with our model and the real one is then maybe of concern for low saturations (Yang et al., 2016).

\section{Conclusion}

In this work, we presented an application to a realistic fracture of a computation method based on two Lattice Boltzmann Models for obtaining of the effective diffusion coefficient $D_{e}$ as a function of saturation $S$.

The results obtained for the particular fracture geometry indicated that the $D_{e}(S)$ curve can be fitted, at first approximation, by a linear Archie's law with a less than $10 \%$ error. In order to better fit the data for low saturation values, a Maxwell expression is obviously more adequate but underestimate the effective diffusion coefficient of about $20 \%$ for saturations around 0.8 . For a more precise fit of the computed data for high saturations, a linear/quadratic curve extracted from the percolation theory with a transition around $S=0.8$ can be used.

The presented methodology appears as a starting point towards a better understanding of the diffusion in argillites fractures on the micrometric scale. It can be used to study, from a statistical point of view, the effective diffusion coefficient as a function of saturation for typical fracture geometries. These geometries can vary from single rough ones similar to the fracture used in this work to more complex interconnected rough fractures structures. Another aspect would consist in coupling the diffusion in fractures with matrix diffusion outside the fracture which would allow to connect the fractures with the isolated pores detected by the micro-tomography.

\section{Acknowledgements}

Acknowledgements The authors acknowledge the financial support of the NEEDS-MIPOR program within "Mission for Interdisciplinarity" of the French National Centre for Scientific Research which partially funded Soukaina Gueddani's graduate engineer internship at IRSN.

\section{References}

Alonso, U., Missana, T., Garcia-Gutierrez M., Patelli, A., Albarran, N., Rigato, V.: Colloid diffusion coefficients in compacted and consolidated clay barriers: Compaction density and colloid size effects. Physics and Chemistry of the Earth 36, 1700-1707 (2011)

Appert, C., Zaleski, S.: Lattice-gas with a liquid-gas transition. Phys. Rev. Lett. 64, 1-4 (1990)

Archie, G.E.: The electrical resistivity $\log$ as an aid in determining some reservoir characteristics. Petroleum Trans. AIME 146, 54-62 (1942)

Balberg, I.: Excluded-volume explanation of Archie's law. Phys. Rev. B 33, 3618-3620 (1986)

Bear, J., Bachmat, Y.: Introduction to modeling of transport phenomena in porous media. Dordrecht, The Netherlands: kluwer Academic Publishers, 1991. 
Bertei, A., Nucci, B., Nicolella, C.: Effective transport properties in random packings of spheres and agglomerates. Chemical Engineering Transactions 32, 1531-1536 (2013)

Boudreau, B.P.: The diffusive tortuosity of fine-grained unlithified sediments. Geochim. Cosmochim. Acta 60(16), 3139-3142 (1996)

van Brakel, J., Heertjes, P.M.: Analysis of diffusion in macroporous media in terms of a porosity, a tortuosity and a constrictivity factor. Int. J. Heat Mass Transfer. 17, 1093-1103 (1974)

Chau, J.F., Or, D., Sukop, M.C.: Simulation of gaseous diffusion in partially saturated porous media under variable gravity with lattice Boltzmann methods. Water Resour. Res. 41, W08410, doi:10.1029/2004WR003821 (2005)

Croisé, J., Mayer, G., Talandier, J., Wendling, J.: Impact of Water Consumption and Saturation-Dependent Corrosion Rate on Hydrogen Generation and Migration from an Intermediate-Level Radioactive Waste Repository. Transp. Porous Med. 90, 59-75 (2011)

Dagnelie, R.V.H., Arnoux, P., Radwan, J., Lebeau, D., Nerfie, P., Beaucaire, C.: Perturbation induced by EDTA on $\mathrm{HDO}, \mathrm{Br}-$ and $\mathrm{Eu}^{I I}$ diffusion in a large-scale clay rock sample. Applied Clay Science 105-106 142-149 (2015)

Delay, J., Vinsot, A., Krieguer, J.M., Rebours, H., and Armand, G.: Making of the underground scientific experimental programme at the Meuse/Haute-Marne underground research laboratory. Physics and Chemistry of the Earth 32, 2-18 (2007)

Epstein, N.: On tortuosity and the tortuosity factor in flow and diffusion through porous media. Chem. Engng. Sci. 44(3), 777-779 (1989)

Friedman, S.P.: Soil properties influencing apparent electrical conductivity: A review. Comput. Electron. Agric. 46, 45-70 (2005)

Genty, A., Pot, V.: Numerical simulation of 3D liquid-gas distribution in porous media by a two-phase TRT lattice Boltzmann method. Transp. Porous Med. 96, 271-294 (2013)

Genty, A., Pot, V.: Numerical Calculation of Effective Diffusion in Unsaturated Porous Media by the TRT Lattice Boltzmann Method Transp. Porous Med. 105, 391-410 (2014)

Ghanbarian, B., Hunt, A.G., Ewing, R.P., Sahimi, M.: Tortuosity in porous media: a critical review. Soil Sci. Soc. Am. J. 77, 1461-1477 (2013)

Ghanbarian, B., Daigle, H., Hunt, A.G., Ewing, R.P., Sahimi, M.: Gas and solute diffusion in partially saturated porous media: Percolation theory and Effective Medium Approximation compared with lattice Boltzmann simulations. J. Geophys. Res. Solid Earth 120, $182-190(2015)$

Ginzburg, I., d'Humières, D.: Multireflection boundary conditions for lattice Boltzmann models. Phys. Rev. E 68(6), 066614 (2003)

Ginzburg, I.: Equilibrium-type and link-type lattice Boltzmann models for generic advection and anisotropic-dispersion equation. Adv. Water Resour. 28, 1171-1195 (2005)

Ginzburg, I., Verhaeghe, F., d'Humières, D.: Study of simple hydrodynamics solutions with the two-relaxation-times lattice Boltzmann scheme. Commun. Comput. Phys. 3, 519-581 (2008)

Hamamoto, S., Moldrup, P., Kawamoto, K., Komatsu, T.: Excluded-volume expansion of Archie's law for gas and solute diffusivities and electrical and thermal conductivities in variably saturated porous media. Water Resour. Res. 46, W06514, doi:10.1029/2009WR008424 (2010)

$\mathrm{Hu}$, Q., Wang, J.S.Y.: Aqueous-phase diffusion in unsaturated geologic media: A review. Critical Reviews in Environmental Science and Technology, 33(3), 275-297 (2003)

d'Humières, D., Ginzburg, I.: Viscosity independent numerical errors for Lattice Boltzmann models: from recurrence equations to "magic" collision numbers. Comp. Math. with Applications 58(5), 823-840 (2009) 
Jones, S.B., Or, D., Bingham, J.E.: Gas Diffusion Measurement and Modeling in CoarseTextured Porous Media. Vadose Zone J. 2, 602-610 (2003)

Marschall, P., Horseman, S., Gimmi, T.: Characterization of Gas Transport Properties of the Opalinus Clay, a Potential Host Rock Formation for Radioactive Waste Disposal. Oil \& Gas Science and Technology - Rev. IFP 60, 121-139 (2005)

de Marsily G.: Quantitative Hydrogeology. San Diego, USA: Academic Press, 1986

Martys, N.S.: Diffusion in partially-saturated porous materials. Mater. Struct. 32, 555-562 (1999)

Martys, N.S., Chen, H.: Simulation of multicomponent fluids in complex three-dimensional geometries by the lattice Boltzmann method. Phys. Rev. E 53(1), 743-750 (1996)

Matray, J-.M., Savoye, S., Cabrera, J.: Desaturation and structure relationships around drifts excavated in the well-compacted Tournemire's argillite (Aveyron, France). Engineering Geology 90(1-2), 1-16 (2007)

Maxwell, J.C.: A treatise on electricity and magnetism. Vol I, Chap IX, Claredon Press, Oxford, 1873

Mayor, J.-C., Velasco, M., García-Siñeriz, J.-L.: Ventilation experiment in the Mont Terri underground laboratory. Physics and Chemistry of the Earth, 32(8-14), 616-628 (2007)

Mercado-Mendoza, H., Lorente, S., Bourbon, X.: The diffusion coefficient of ionic species through unsaturated materials. Transp. Porous Med. 96, 469-481 (2013)

Moldrup, P., Olesen, T., Yoshikawa, S., Komatsu, T., Rolston, D.E.: Three-porosity model for predicting the gas diffusion coefficient in unsaturated soil. Soil Sci. Soc. Am. J. 68, $750-759$ (2004)

Nakashima, Y., Nakano, T.: Steady-state local diffusive fluxes in porous geo-materials obtained by pore seale simulations. Transp. Porous Med. 93, 657-673 (2012)

Patriarche, D., Michelot, J.-L., Ledoux, E., Savoye, S.: Diffusion as the main process for mass transport in very low water content argillites: 1 . Chloride as a natural tracer for mass transport - Diffusion coefficient and concentration measurements in interstitial water. Water Resour. Res., 40, W01516, doi:10.1029/2003WR002600.

Poller, A., Enssle, C.P., Mayer, G., Croisé, J., Wendling, J.: Repository-Scale Modeling of the Long-Term Hydraulic Perturbation Induced by Gas and Heat Generation in a Geological Repository for High-and Intermediate-Level Radioactive Waste: Methodology and Example of Application. Transp. Porous Med. 90, 77-94 (2011)

Pot, V., Hammou, H., Elyeznasmi, N., Ginzburg, I.: Role of soil heterogeneities onto pesticide fate: a pore-scale study with lattice Boltzmann. in Proc. of the 1st international conference and exploratory workshop on soil architexture and physico-chemical functions "CESAR", ISBN 87 91949-59-9, Nov. 30 - Dec. 2, 2010, Research Centre Foulum, Tjele, Denmark

Pot, V., Peth, S., Monga, O., Vogel, L.E., Genty, A., Garnier, P., Vieublé-Gonod, L., Ogurreck, M., Beckmann, F., Baveye, P.C.: Three-dimensional distribution of water and air in soil pores: Comparison of two-phase two-relaxation-times lattice-Boltzmann and morphological model outputs with synchrotron X-ray computed tomography data. Advances in water Resources 84, 87-102 (2015)

Raiskinmäki, P., Koponen, A., Merikoski, J., Timonen, J.: Spreading dynamies of threedimensional droplets by the lattice-Boltzmann method. Comp. Mater. Sci. 18, 7-12 (2000)

Rübel, A.P., Sonntag, C., Lippmann, J., Pearson, F.J., Gautschi, A.A.: Solute transport in formations of very low permeability: Profiles of stable isotope and dissolved noble gas contents of pore water in the Opalinus Clay, Mont Terri, Switzerland. Geochimica Cosmochimica Acta 66(8), 1311-1321 (2002) 
Saripalli, K.P., Serne, R.J., Meyer, P.D., McGrail, B.P.: Prediction of diffusion coefficients in porous media using tortuosity factors based on interfacial areas. Ground Water $40(4)$, 346-352 (2002)

Savoye, S., Page, J., Puente, C., Imbert, C., Coelho, D.: New experimental approach for studying diffusion through an intact and unsaturated medium: a case study with CallovoOxfordian argillite. Environ. Sci. Technol. 44(10), 3698-3704 (2010)

Shan, X., Chen, H.: Lattice Boltzmann model for simulating flows with multiple phases and components. Phys. Rev. E 47(3), 1815-1820 (1993)

Shan, X., Chen, H.: Simulation of nonideal gases and liquid-gas phase transitions by the lattice Boltzmann equation. Phys. Rev. E 49(4), 2941-294 (1994)

Shen, L., Chen, Z.: Critical review of the impact of tortuosity on diffusion. Chemical Engineering Sci. 62, 3748-3755 (2007)

Voutilainen, M., Sardini, P., Siitari-Kauppi, M., Kekaäläinen, P., Aho, V., yllys, M., Timonen, J.: Diffusion of tracer in altered tonalite: experiments and simulations with heterogeneous distribution of porosity. Transp. Porous Med. 96, 319-336 (2013)

$\mathrm{Xu}, \mathrm{T}$., Senger, R., Finsterle, S.: Corrosion-induced hydrogen generation in a nuclear waste repository: reactive geochemistry and multiphase flow effects. Appl. Geochem. 23, 34233433 (2008)

Xuan, Y.M., Zhao, K., Li, Q.: Investigation on mass diffusion process in porous media based on Lattice Boltzmann method. Int. J. Heat Mass Transf. 46, 1039-1051 (2010)

Yang, R., Lemarchand, E., Fen-Chong, T.: A Micromechanics Model for Solute Diffusion Coefficient in Unsaturated Granular Materials. Transp. Porous Med. 111, 347-368 (2016)

Zhang, M., Ye, G., van Breugel, K.: Modeling of ionic diffusivity in non-saturated cementbased materials using lattice Boltzmann method. Cem. Concr. Res. 42(11), 1524-1533 (2012) 